\title{
Divergent RNA binding specificity of yeast Puf2p
}

\author{
YAHAV YOSEFZON, ${ }^{1}$ YVONNE Y. KOH, ${ }^{2}$ JACQUELINE J. CHRITTON, ${ }^{2}$ AVIGAIL LANDE, ${ }^{1}$ LIMOR LEIBOVICH, ${ }^{3}$ \\ LAVI BARZIV, ${ }^{1}$ CHRISTINE PETZOLD, ${ }^{2}$ ZOHAR YAKHINI, ${ }^{3,4}$ YAEL MANDEL-GUTFREUND, ${ }^{1}$ \\ MARVIN WICKENS, ${ }^{2,5}$ and YOAV ARAVA ${ }^{1,5}$ \\ ${ }^{1}$ Department of Biology, Technion-Israel Institute of Technology, Haifa 32000, Israel \\ ${ }^{2}$ Department of Biochemistry, University of Wisconsin-Madison, Madison, Wisconsin 53706, USA \\ ${ }^{3}$ Department of Computer Science, Technion-Israel Institute of Technology, Haifa 32000, Israel \\ ${ }^{4}$ Agilent Laboratories Israel, Petach-Tikva 49527, Israel
}

\begin{abstract}
PUF proteins bind mRNAs and regulate their translation, stability, and localization. Each PUF protein binds a selective group of mRNAs, enabling their coordinate control. We focus here on the specificity of Puf2p and Puf1p of Saccharomyces cerevisiae, which copurify with overlapping groups of mRNAs. We applied an RNA-adapted version of the DRIM algorithm to identify putative binding sequences for both proteins. We first identified a novel motif in the $3^{\prime}$ UTRs of mRNAs previously shown to associate with Puf2p. This motif consisted of two UAAU tetranucleotides separated by a 3-nt linker sequence, which we refer to as the dual UAAU motif. The dual UAAU motif was necessary for binding to Puf2p, as judged by gel shift, yeast three-hybrid, and coimmunoprecipitation from yeast lysates. The UAAU tetranucleotides are required for optimal binding, while the identity and length of the linker sequences are less critical. Puf1p also binds the dual UAAU sequence, consistent with the prior observation that it associates with similar populations of mRNAs. In contrast, three other canonical yeast PUF proteins fail to bind the Puf $2 p$ recognition site. The dual UAAU motif is distinct from previously known PUF protein binding sites, which invariably possess a UGU trinucleotide. This study expands the repertoire of cis elements bound by PUF proteins and suggests new modes by which PUF proteins recognize their mRNA targets.
\end{abstract}

Keywords: Puf proteins; RNA binding proteins; PUM domain

\section{INTRODUCTION}

RNA binding proteins (RBPs) control the amount and nature of proteins produced from a gene. In the nucleus, they regulate RNA processing and export in the cytoplasm, they control mRNA stability, translation, and localization (Scherrer et al. 2010; Tsvetanova et al. 2010). A single RBP can bind and control tens to hundreds of functionally related mRNAs (Keene and Tenenbaum 2002; Hieronymus and Silver 2003; Gerber et al. 2004; Hogan et al. 2008; Kanitz and Gerber 2010; Kershner and Kimble 2010), enabling one protein to coordinately control many mRNAs underlying a biological event.

PUF proteins, named after the two founding members, Drosophila Pumilio and Caenorhabditis elegans fem-3 binding factor (FBF) (Zamore et al. 1997; Zhang et al. 1997), exemplify this coordinate control. Members of the PUF family contain a PUF repeat region (or PUM-homology domain, PUM-HD),

\footnotetext{
${ }^{5}$ Corresponding authors.

E-mail wickens@biochem.wisc.edu.

E-mail arava@tx.technion.ac.il.

Article published online ahead of print. Article and publication date are at http://www.rnajournal.org/cgi/doi/10.1261/rna.2700311.
}

which usually comprises eight tandem PUF repeats, each containing about 40 amino acids. This domain is sufficient for RNA binding and interacts with protein coregulators (e.g., Kraemer et al. 1999; Sonoda and Wharton 1999). PUF proteins regulate mRNA expression by binding to short sequences in the 3' UTR of their target mRNAs and recruiting other proteins that promote mRNA translational repression, degradation, and localization. The binding sites of several PUF proteins have been identified biochemically and through informatics (Gerber et al. 2004; Bernstein et al. 2005; Opperman et al. 2005; Galgano et al. 2008; Hogan et al. 2008; Morris et al. 2008; Stumpf et al. 2008a; Koh et al. 2009). To date, all binding sites that have been confirmed experimentally comprise 8-11 nt and include a UGUR tetranucleotide core motif.

Crystal structures of PUM domains reveal common PUF scaffolds and diverse strategies for achieving RNA specificity (Edwards et al. 2001; Wang et al. 2001; Miller et al. 2008; Wang et al. 2009; Zhu et al. 2009). The overall structures of human Pumilio, C. elegans FBF, and Saccharomyces cerevisiae Puf3p and Puf4p bound to RNA are very similar. Invariably, each PUF repeat folds into a three-helix domain; these domains stack on one another to form a crescent. The 
crescent provides two extended surfaces: an inner one that contacts RNA, and an outer one that may interact with proteins (Wickens et al. 2002). In the simplest case, as exemplified by human Pumilio, each PUF repeat recognizes a single RNA base through three conserved side chains (Wang et al. 2002). The RNA runs antiparallel to the protein such that nucleotides 1-8 are recognized individually by PUF repeats 81 , respectively. This mode of recognition, in which one PUF repeat interacts with one RNA base, provides a simple RNAprotein scaffold (Wang et al. 2002). Despite their common overall architectures, not all PUF proteins bind the same sequence. In all examples studied to date, PUF repeats 6-8 bind UGU. Specificity arises elsewhere, through two structural principles: base flipping and specific binding pockets. In base flipping, a local region of the protein imposes the requirement for an additional base in the recognition site, flipping that base out away from the protein (Wang et al. 2009). Flipping occurs at different locations in C. elegans FBF, yeast Puf4p, and human Pumilio bound to a noncognate site (Gupta et al. 2008; Miller et al. 2008; Wang et al. 2009). The second contributor to specificity comprises a distinctive binding pocket for a nucleotide upstream of UGU, as described for yeast Puf3p (Zhu et al. 2009).

A single species often contains multiple PUF proteins, each with a distinctive set of targets. S. cerevisiae cells contain six PUF proteins, designated Puflp to Puf6p. Each protein associates with many mRNAs, suggesting that nearly $10 \%-$ $15 \%$ of yeast mRNAs are regulated by the PUF family (Gerber et al. 2004). The mRNAs that bind each protein are largely distinct. For example, Puf3p binds nearly exclusively cytoplasmic mRNAs that encode mitochondrial proteins, and Puf4p and Puf5p interact selectively with mRNAs encoding nuclear components (Gerber et al. 2004). Puf2p and Puflp interact preferentially with mRNAs encoding membraneassociated proteins (Gerber et al. 2004). Puf1p, Puf3p, Puf4p, and Puf5p enhance mRNA degradation (Hook et al. 2007; Ulbricht and Olivas 2008). Puf3p is involved in localization of mRNAs (Saint-Georges et al. 2008; Eliyahu et al. 2010), as is Puf6p. Puf6p represses translation of the ASH1 mRNA (Gu et al. 2004; Deng et al. 2008). Thus, it appears that the yeast PUF members have distinct roles in post-transcriptional regulation that are exerted on specific sets of mRNAs.

In this study, we focus on Puf2p of $S$. cerevisiae. We used an advanced computational approach to search for a Puf2p target motif. We identified a novel motif, which differs from the canonical UGUR motif and appears more significant than a previously suggested motif (Hogan et al. 2008). This motif is composed of two UAAU tetranucleotides, separated by a 3-nt linker. We show that Puf2p binds this element in vitro and in vivo and that both UAAU subdomains are important for binding. Altering this element in a natural target of Puf2p led to a reduction in its association with Puf2p. Puflp recognizes this sequence element, while other PUF proteins do not. We suggest that Puf2p exploits a different mode of RNA recognition from other members of the PUF family.

\section{RESULTS}

\section{Prediction of Puf2p binding site}

A genome-wide affinity purification study identified mRNAs that were associated with Puf2p in yeast extracts (Gerber et al. 2004). While originally no consensus motif was found among the associated RNAs (Gerber et al. 2004), Hogan et al. (2008) applied a MEME motif-search algorithm (Bailey et al. 2009) and detected a new motif for Puf2p, UAAUAAUUW.

We sought to characterize the Puf2p binding site using the DRIM algorithm (discovery of rank imbalanced motifs; see Materials and Methods) (Eden et al. 2007). An advantage of DRIM in the analysis of microarray data is that it does not require prior selection of the affected targets and therefore can be applied to an entire data set without defining an arbitrary threshold of enrichment.

We applied the algorithm to the original data set, seeking 3' UTR motifs that were most enriched in the immunopurification with Puf2p (Gerber et al. 2004). The most significant motif $\left(P\right.$-value $\left.<10^{-48}\right)$ found among RNAs associated with Puf2 $p$ included two UAAU tetranucleotides separated by a 3-nt linker (Fig. 1A). This motif was also enriched in the $5^{\prime}$ UTRs and the coding regions, but to a lesser extent $(P$-values of $2 \times 10^{-6}$ and $3 \times 10^{-10}$, respectively). To examine
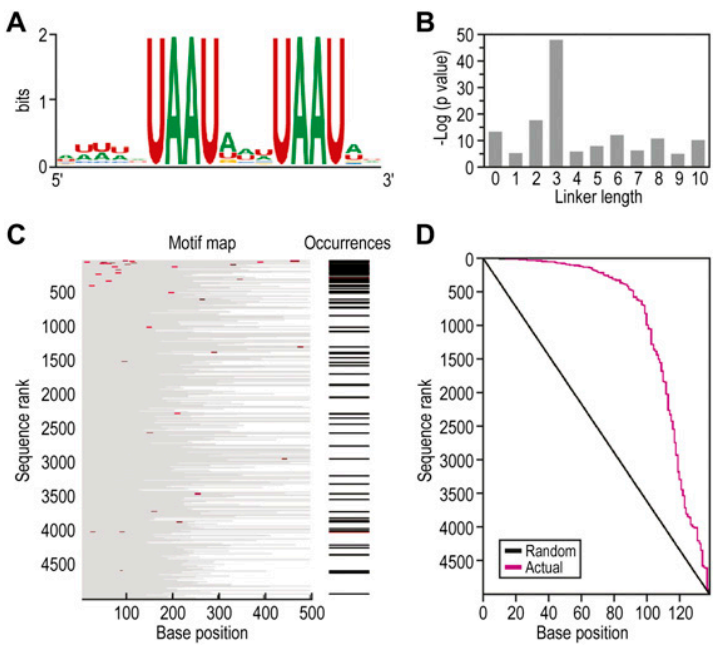

FIGURE 1. DRIM-predicted Puf2 binding sequence. $(A)$ Sequence of the most enriched motif among Puf2p targets presented in a Logo format. (B) Variants of the most enriched motif were compared, each consisting of two UAAU half-sites connected with a linker of a variable length (0 to $10 \mathrm{nt})$. (C) Motif map of the predicted motif. All of the 4997 mRNAs that were detected in the microarray experiment of Puf2 purification in Gerber et al. (2004) were ranked according to the obtained signal, and their 3' UTRs are presented as gray lines. The lengths of the gray lines are proportional to the $3^{\prime}$ UTRs' lengths (Nagalakshmi et al. 2008). (Red) The motif position along the $3^{\prime}$ UTR. The "Occurrence" column indicates the number of motif occurrences in each $3^{\prime}$ UTR sequence (black for one occurrence and red for two occurrences). (D) Accumulation graph relative to a random model. (Red line) The actual motif accumulated occurrences in relation to the sequences rank; (black line) the expected occurrences. 
whether the length of the linker between the two UAAU elements was conserved among the 3' UTRs, we calculated the statistical enrichment of different linker lengths. All variants contained the two core sites separated by a linker of variable length, from a range of 0 to $10 \mathrm{nt}$ (Fig. 1B). A 3-nt linker was most prevalent among Puf2p targets.

Figure $1 \mathrm{C}$ provides a visual rendering of the DRIM output: 4997 genes passed the quality selection criteria in the published microarray experiment (Gerber et al. 2004). The 3' UTRs of these mRNAs are depicted as gray lines (proportional to their lengths) and are ordered along the $y$-axis according to their enrichment among Puf2p-associated mRNAs. Occurrences of UAAUNNNUAAU motifs within these $3^{\prime}$ UTRs are indicated in red, positioned by distance from the termination codon along the $x$-axis. The entire list of genes and the position of the motif (also for the 5' UTR and the coding regions) appear in Supplemental Table 1. The putative Puf2p binding motif is highly enriched among those mRNAs that are ranked high in the list. Optimum enrichment of this motif was attained among the top 336 genes: 69 contained the motif in their $3^{\prime}$ UTRs. Note that in several of the sequences that appear in the top of the list, the consensus motif occurs multiple times in a single $3^{\prime}$ UTR. Among the 69 targets that contained the motif, eight of the targets contained two motifs, and a single target contained four. The statistical significance of the enrichment is visible in Figure 1D, where the motif accumulated occurrences (pink) are plotted versus the expected accumulated occurrences in a random data set (black). While in a random data set the occurrences are distributed uniformly (hence a diagonal line), in the actual data set the UAAU motif is enriched in the $3^{\prime}$ UTRs at the top of the list. We refer to the UAAUNN NUAAU sequence as the "dual UAAU" motif.

\section{In vitro validation of Puf2 predicted motif}

To test binding of Puf2p to the predicted motif experimentally, we performed electrophoretic mobility shift assays (EMSA). In this assay, we combined increasing concentrations of purified GST-Puf2p PUM domain (Fig. 2A) with a 40-nt RNA derived from the PMP2 3' UTR that contains the dual UAAU motif (Fig. 2B,E). Puf2p-PUM domain bound the PMP2 RNA with an apparent $K_{\mathrm{d}}$ of $53 \pm 6 \mathrm{nM}$ (Table 1), in the range of other PUF proteins
(Bernstein et al. 2005; Koh et al. 2009). This binding is not due to the GST moiety because the GST-Puf5p fusion protein did not bind the dual UAAU motif (see Fig. 6 below). Moreover, partial purification of Puf2p PUM domain (after cleavage of its GST moiety) did not change its apparent $K_{\mathrm{d}}$ (62 nM) (data not shown).

To simplify identification of the minimal binding element in the PMP2 RNA, we created a second RNA oligo that disrupts an adjacent UAAU sequence and shortens the RNA to $30 \mathrm{nt}$. This RNA is referred to as $P M P 2^{\star}$. Puf2p bound the $P_{M P 2}{ }^{*}$ RNA with the same affinity as the original $40-n t$ PMP2 RNA (Fig. 2C,F). Furthermore, when the consensus UAAUNNNUAAU motif was replaced with AC repeats, affinity decreased significantly (apparent $K_{\mathrm{d}}$ of $354 \mathrm{nM}$ ) (Fig. 2D,F; Table 1).

In immunopurification studies, Puf2p associated with many mRNAs that do not contain the dual UAAU motif (Gerber et al. 2004). One such mRNA is PMP1, which contains a variation of the dual UAAU motif that converts the second UAAU to UAAAU (Table 1). This "deviant" 
TABLE 1. Summary of in vitro binding assay results

\begin{tabular}{|c|c|c|}
\hline RNA & Sequence of Oligo & $K_{d}$ \\
\hline PMP2 & UUUAAUUUCUAAUAAUUAAUUAAUUUUUCCUGUGUGUGUG & $53 \pm 6(n=4)$ \\
\hline PMP2* & AAUUUCUAAUAAUUAAUACAUUUUUCCUCU & $54 \pm 8(n=3)$ \\
\hline PMP2* Mut. & AAUUUCCacacacacaaacAUUUUUCCUCU & $354 \pm 57(n=3)$ \\
\hline PMP2* 1 Linker & AAUUUCUAAUUUAAUACAUUUUUCCUGUGUGUGUG & $170 \pm 32(n=2)$ \\
\hline PMP2* 6 Linker & AAUUUCUAAUauauauUAAUaCAUUUUUCCUGUGUGUGUG & $57 \pm 16(n=2)$ \\
\hline PMP1 & AGAAAAUCUAAAUAAUAAAUAAAUAAAAAA & $111 \pm 11(n=4)$ \\
\hline PMP1 Mut. & AGAAAAUCUAAAUgugugugAAAUAAAAAA & $515 \pm 56(n=2)$ \\
\hline
\end{tabular}

The indicated oligos were subjected to EMSA with increasing concentrations of purified Puf $2 p$ PUM domain, and $K_{d}$ values were calculated as in Figure 2. The dual UAAU motif is shaded in gray, and mutated bases are in lowercase.

sequence still bound Puf2p in vitro, albeit with a twofold reduced apparent $K_{\mathrm{d}}(111 \mathrm{nM})$. The affinity was further reduced fivefold by mutations that disrupted both UAAU elements (Table 1), indicating that binding to the PMP1 sequence was specific.

\section{Analysis of association with TAP-Puf2}

To test whether the dual UAAU motif was important for binding of Puf $2 p$ to endogenous mRNAs, we generated two yeast strains: one that expresses the wild-type (WT) PMP2 transcript and one that expresses a mutant version of the dual UAAU motif (Fig. 3A). Both strains also expressed TAP-tagged Puf2p. Association of the PMP2 transcript with the TAP-tagged Puf2p was assayed by affinity purification and Northern blot analysis (Fig. 3B). As a negative control, the same blots were hybridized with a probe to an mRNA that is not associated with Puf2 (ACT1) (Gerber et al. 2004). No signal was detected in the purified samples even after longer exposures of the blot, excluding nonspecific binding. Furthermore, to normalize for differences between the strains, the Northern blots were also probed for a second Puf2p-associated mRNA, PMP3 (Gerber et al. 2004). A reproducible $\sim 40 \%$ decrease in association of the mutated transcript with Puf2p compared with the WT PMP2 construct was observed (Fig. 3B). This confirms that the dual UAAU motif contributes to Puf2p association in vivo.

\section{Puf2p interactions in vivo}

To further define the Puf2p binding site, we used the yeast three-hybrid system (Stumpf et al. 2008b). In this analysis, the interaction between the Puf2p-Gal4 activation domain fusion protein and an RNA sequence results in activation of a $L a c Z$ reporter (Fig. $4 \mathrm{~A}$ ). LacZ enzyme activity in this assay correlates with RNA-protein affinity as measured in vitro over a 10 -fold to 100 -fold range (Hook et al. 2005).

We first tested a 27-nt RNA sequence derived from the PMP2 3' UTR containing the dual UAAU motif. The combination of the PMP2 RNA and Puf2p resulted in activation of the LacZ reporter, while the vector control RNA did not
(Fig. 4B). We again eliminated the complication of the additional UAAU by introducing two point mutations, converting that UAAU sequence to ACAU (Fig. 4B). This RNA is referred to as $P M P 2^{*}$. The resulting RNA bound well and provided a template for all future mutagenesis studies. Transversion mutations in $P M 2^{\star}$ in the upstream UAAU (mut1), the downstream UAAU (mut2), or both (mut1-2) eliminated binding (Fig. 4B). We conclude that both UAAU elements are important for the interaction with Puf2p.

To identify the minimal segment of RNA capable of binding well to Puf2p, we prepared RNAs in which all the nucleotides flanking the two UAAU sequences were converted to alternating CA repeats (Fig. 4C). A segment of RNA containing only UAAUAAUUAAU derived from $P M P 2$, flanked by repeating CA sequences, bound as well as the WT sequence. In that background, mutation of a single $U$ from either the $5^{\prime}$ or $3^{\prime}$ end reduced binding modestly; removal of a nucleotide from both ends simultaneously reduced binding to undetectable levels. We conclude that the minimal sequence required for high-affinity binding is the 11-nt sequence UAAUAAUUAAU.

To analyze the effect of the sequence between the two UAAU elements - the "linker"-we tested RNAs in which the spacing was varied from 0 to $6 \mathrm{nt}$ (Fig. 4D). All of the RNAs bound with either WT or slightly reduced affinity. We also varied the identity of the nucleotides in the linker. While, in most cases, these changes did not affect binding, an $\sim 20$-fold difference was observed between the WT,

A

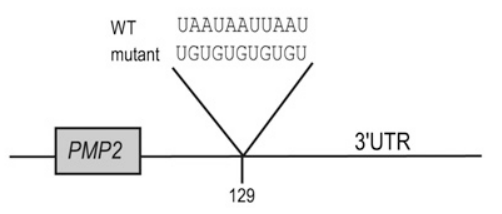

B
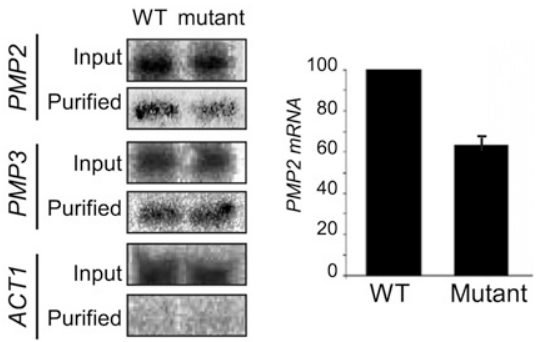

FIGURE 3. Mutation of the dual UAAU motif reduces Puf2p binding in vivo. (A) Yeast strains expressing TAP-tagged Puf2 $p$ and either the wild-type (WT) or mutant (mut) PMP2 mRNA (depicted schematically) were subjected to affinity purification with Protein A-Sepharose beads. (B) Equal amounts of RNA prior to purification (Input) and after purification (Purified) from the two strains were analyzed by Northern blotting with a probe for PMP2 mRNA. To account for variation during the purification procedure, the membranes were also probed for unmodified Puf2p-associated mRNA (PMP3). Hybridization to a non-Puf2 target (ACT1) was performed to exclude nonspecific binding to the beads. The graph shows the results of PMP2 normalized to the signals of PMP3 from three independent experiments. 
A

B

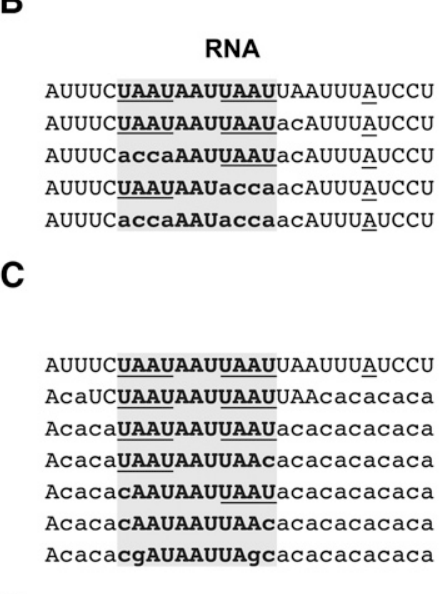

D

AUUUCUAAU------UAAUaCAUUUA AUUUCUAAUa------UAAU CACAUUA AUCCU

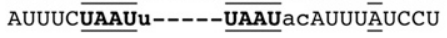
AUUUCUAAUau----- UAAUUCAUUUAUCCU AUUUCUAAUaau---UAAUACAUUUAUCCU (PMP2*) AUUUCUAAUU CCa--- $\underline{\text { UAAUU }}$ acAUUUĀUCCU AUUUCUAAUauau--UAAUACAUUUAUCCU AUUUCUAAU acau-- UAAU $a c A U U U$ A-UCCU AUUUCUAAUaaCaU-UAAUACAUUUAUCCU AUUUCUAAU accau-UAAU AUUUCUAAUauauauUAAUaCAUUUAUCCU AUUUCUAAU acacau UAAUUaCAUUUA UACCU
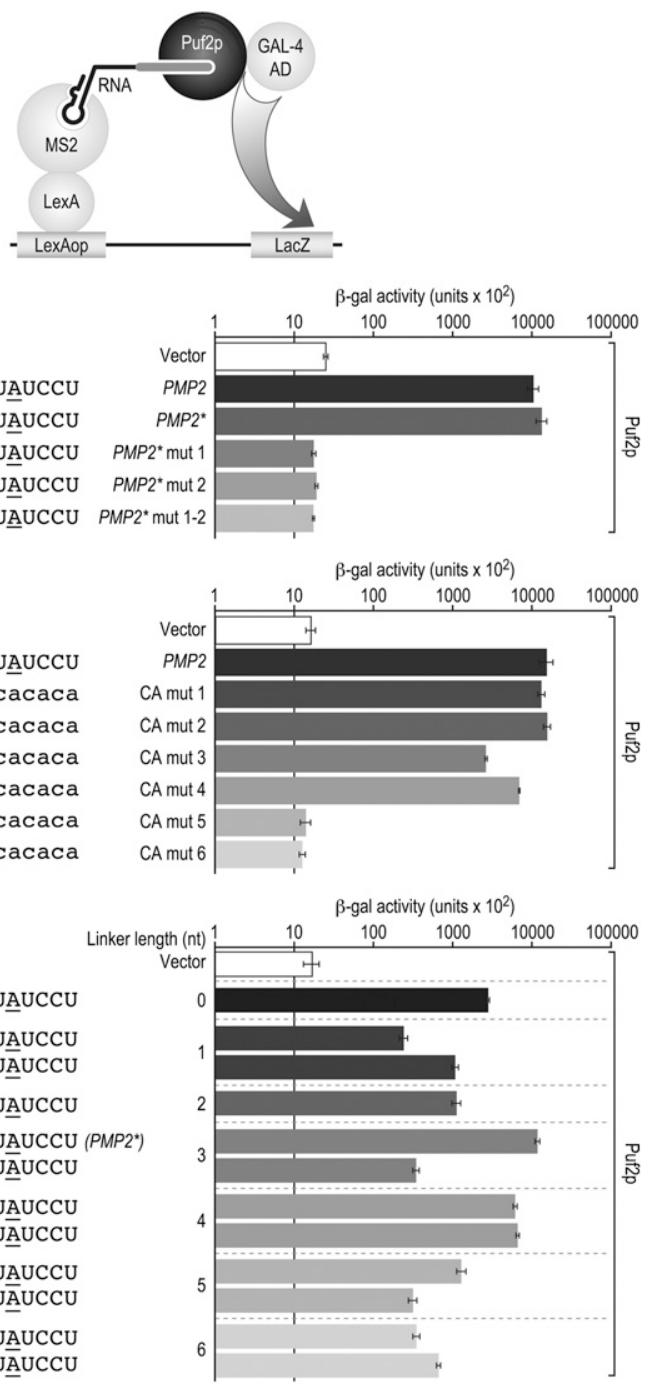

FIGURE 4. The minimal binding site for Puf2p. (A) The yeast three-hybrid assay. The assay is used to detect interactions between a specific RNA-protein pair. An interaction between the target protein, in our case Puf2p, and the target RNA triggers expression of the reporter gene $L a c Z$, which can be quantitated to determine the relative strength of the interaction. $(B)$ Yeast three-hybrid assay with Puf2p and the RNA sequences shown on the left. Bases in lowercase are mutations in the PMP2 sequence. An underlined base indicates a U-to-A substitution to prevent five consecutive uridines (see Materials and Methods). The core dual UAAU motif is highlighted in gray. $(C)$ The $P M P 2$ sequence was sequentially mutated to CA repeats (lowercase) on either side to determine the minimal sequence required to bind Puf2p. The minimal sequence for maximal Puf2p binding was UAAUAAUUAAU. (D) The effect of different linker lengths and compositions on Puf2p binding was analyzed. The linker was defined as the sequence between the two UAAU elements. $P M P 2^{*}$ containing the wildtype linker sequence (AAU) is indicated. The linker length is indicated to the left of the bar graph.

AAU linker to the modified CCA linker (Fig. 4D). The higher affinity of the motif with an AAU linker may be due to the fact that it also contains a dual UAAU motif without a linker; a motif that Puf2 can bind efficiently. These threehybrid results indicate that Puf2 can efficiently bind motifs with varying lengths of linker. To assess whether this also was true in vitro, we performed EMSAs with PMP2 RNAs of variable linker lengths. We observed a threefold drop in affinity with a 1-nt linker, while a 6-nt linker had no effect on binding (Table 1). We conclude that the presence of the linker is relatively unimportant for binding and that a range of linker lengths and sequences support the interaction with Puf2p.

To test whether binding of Puf2p to the dual UAAU motif requires the same amino acids used in other PUF-RNA interactions, we analyzed mutations in several PUF repeats. Specifically, two conserved residues that contact the RNA in other PUF proteins were mutated to alanine in either two or three adjacent PUF repeats (R1/R2 and R1/R2/R3) (Fig. $5 \mathrm{~A})$. The mutant proteins were expressed at similar levels as WT Puf2p yet did not interact with any RNA tested (Fig. 5A,B). We conclude that the same residues that contact RNA in other PUF proteins likely do so in Puf2p.

In addition to the PUM domain, Puf2p contains a predicted RNA recognition motif (RRM) between amino acids 315 and 397. We tested whether this RRM contributes to the specific binding to the dual UAAU motif, by analyzing binding of an in-frame deletion of the RRM. The RRM deletion protein exhibited a similar binding profile as WT Puf2p (cf. Figs. 4B and 5C). We conclude that the RRM does not dramatically affect the RNA-binding specificity of Puf2p to the dual UAAU motif.

\section{Puf1p and Puf2p specificities are distinct from those of other PUF proteins}

To compare the RNA-binding specificity of Puf2p to those of other PUF proteins, we tested whether Puf2p bound the sites of other PUF proteins, and conversely, whether the dual UAAU motif bound other PUF proteins. Puf $2 p$ did not bind the Puf3p site (COX17 site B) or the Puf4p and Puf5p sites in the HO 3' UTR in the three-hybrid assay (Fig. 6A). Conversely, Puf3p, Puf4p, and Puf5p failed to bind the dual UAAU motif, but did bind their own cognate sites. We further tested selectivity biochemically using EMSA with yeast Puf5p. Purified Puf5p (the PUM domain) failed to bind detectably to the PMP2 RNA, even at protein concentrations as high as $1000 \mathrm{nM}$ (Fig. 6B). In contrast, 
A

AUUUCUAAUAAUUAaUUAAUUUAUUCCU AUUUCUAAUAAUUAAU UCAUUUA UCCU AUUUCACCAAAUUAAU

B

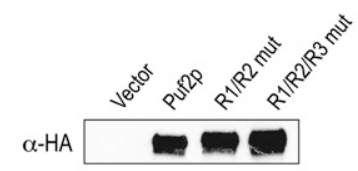

C

RNA

AUUUCUAAUAAUUAAUUAAUUUAUCCU AUUUCUAAUAAUUAAU AUUUCACCAAAUUAAU AUUUCUAAUAAUACCaACAUUUA AUUUCACCaAAUaCCaACAUUUAUUCCU
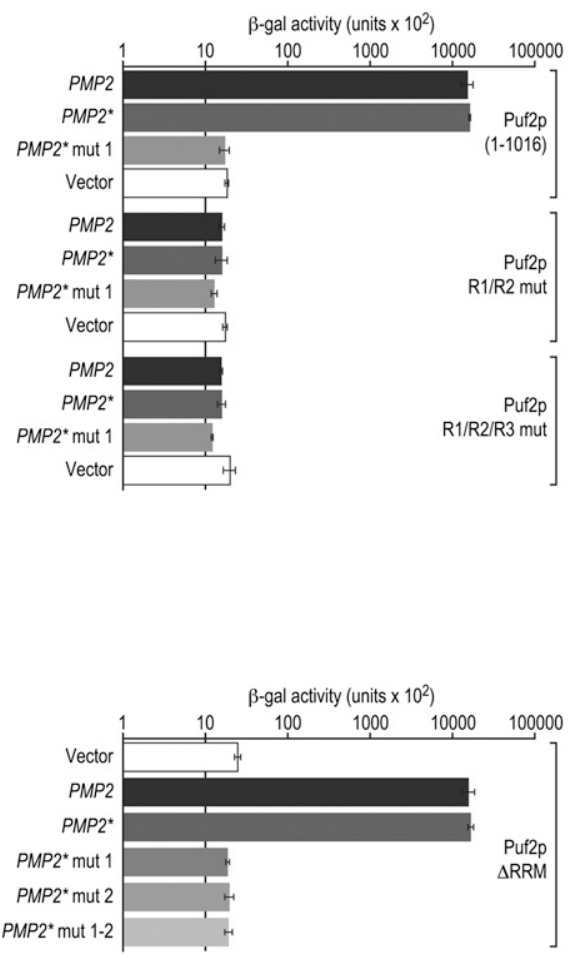

FIGURE 5. The PUM domain in Puf2p confers RNA binding activity. (A) Key residues in PUF repeats that are critical for binding (either the first two repeats [R1/R2 mut] or the first three $[\mathrm{R} 1 / \mathrm{R} 2 / \mathrm{R} 3 \mathrm{mut}]$ ) were mutated and analyzed in the yeast three-hybrid assay. (B) Yeast expressing HA-tagged variants of Puf $2 p$ (from $A$ ) were grown to log phase and processed to obtain yeast extracts. The yeast extracts were analyzed by Western blot using anti-HA antibodies. All proteins are expressed at similar levels. (C) A Puf2p mutant protein lacking the RRM (amino acids 315-397) was analyzed for binding with various RNAs as in Figure 4B. Deletion of the RRM did not affect Puf2p's ability to bind RNA.

Puf5p efficiently binds its own site (in $H O$ ) already at $80 \mathrm{nM}$ (Fig. 6B).

Interestingly, analysis of the binding specificity of the five canonical PUF proteins revealed that Puflp and Puf2p behave similarly; Puflp efficiently bound the dual UAAU motif but not the Puf3p and Puf4p/ Puf5p motifs (Fig. 6A). Their shared binding specificity is consistent with the fact that yeast Puflp and Puf2p share a high degree of sequence relatedness (35\% identity and 50\% similarity), and both interact preferentially with mRNAs encoding membrane-associated proteins (Gerber et al. 2004). DRIM analysis of Puflp-bound RNAs revealed that they too were enriched in the sequence UAAUNNNUAAU (Supplemental Fig. 1A). A linker length of 3 nt was preferred (Supplemental Fig. 1B), as observed with Puf2p-associated RNAs.

To determine whether the yeast Puflp binding profile was similar to that of Puf2p, we assayed Puf1p binding

A

B to select RNAs in the three-hybrid system. Puflp bound PMP2 and PMP2* with high affinity (Fig. 7). As with Puf2p, binding of Puflp to the $P M P 2^{*}$ RNA was eliminated when either one or both UAAU motifs were mutated. Taken together, we conclude that Puflp and Puf2p have a similar binding specificity that is distinct from that of other PUF proteins.

\section{DISCUSSION}

The DRIM algorithm, which searches for common motifs in a ranked list of genes, predicted a novel sequence motif that is significantly enriched in the $3^{\prime}$ UTRs of mRNAs bound by Puf2p (Fig. 1). This motif, designated the dual UAAU motif, is comprised of two tetranucleotides (UAAU) separated by a 3-nt linker. In vitro and in vivo assays revealed that the PUM domain of Puf $2 p$ binds the dual UAAU motif directly and that mutating either UAAU motif leads to a significantly lower interaction, while the distance and the type of bases between them lead to a weaker effect. These empirical measurements yield the consensus $\mathrm{UAAUN}_{0-6} \mathrm{UAAU}$, which is different from the canonical PUF binding site $\left(\mathrm{UGURN}_{1-4} \mathrm{UA}\right)$.

Binding of the PMP1 RNA, which contains a 1-nt addition compared with the consensus motif, is reduced twofold. However, this RNA appears to associate with
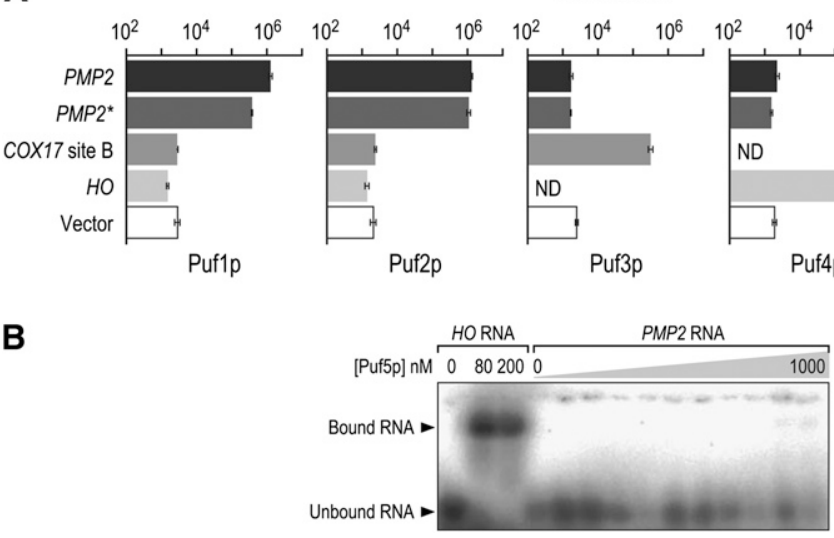

FIGURE 6. Yeast PUF proteins have different binding specificities. $(A)$ The five canonical yeast PUF proteins were combined with representative target RNAs from each PUF in the three-hybrid assay. Puf4p and Puf5p recognize separate sites in the HO $3^{\prime}$ UTR, and Puf3p recognizes a site in the COX17 3' UTR (Olivas and Parker 2000; Hook et al. 2007). (B) PMP2 was subjected to EMSA with increasing concentrations of purified GST-PUM domain of Puf5p. The HO RNA oligo was included as a positive control. 


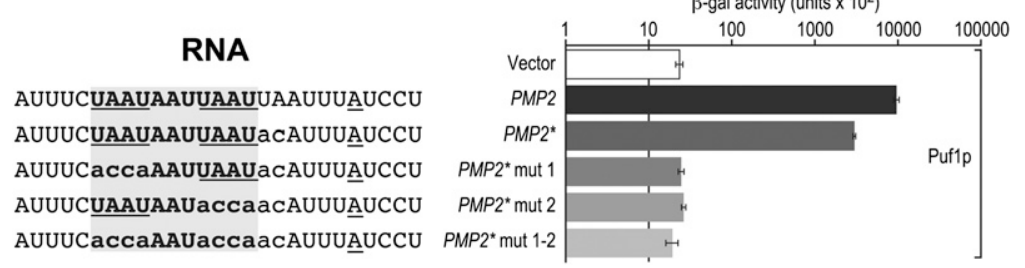

FIGURE 7. Puflp binds the dual UAAU motif. Puflp was analyzed for binding with various RNAs as in Figure 4B.

Puf2p in vivo (Gerber et al. 2004). We infer that variants of the UAAU motif may be bound in vivo, broadening the spectrum of RNAs controlled by Puf2p. A related motif (UAAUAAAUW) was predicted for Puf2p by Hogan et al. (2008) using a different computational method. While the enrichment of the dual UAAU motif is more significant (mHG $P$-value of $10^{-21}$ ), we cannot exclude the possibility that the UAAUAAAUW motif is also functional in vivo, thereby further increasing the complexity of Puf2-RNA interactions. Moreover, Puf2 (and Puf1) have a predicted RRM in addition to the PUM domain. Our results indicate that this domain is not important for binding of Puf2 to the dual UAAU motif. However, considering the broad and significant involvement of RRMs in RNA metabolism (Clery et al. 2008), this motif is likely to further expand the targets of Puf2 by binding to yet-to-determined sequences.

Puf2p diverges from the simplest condition, exemplified by human Pumilio, in which one PUF repeat interacts with one RNA base (Wang et al. 2001). Our results with Puf2p emphasize the diversity of PUF recognition in two ways. First, the binding site does not possess the UGUR sequence seen in all known targets of PUF proteins. Second, Puf2p recognizes $11 \mathrm{nt}$, while the PUM domain of Puf2p contains only six recognizable PUF repeats (Gerber et al. 2004). Other PUF proteins with eight canonical repeats recognize longer binding sites, either due to base flipping or additional nucleotide binding pockets (Bernstein et al. 2005; Opperman et al. 2005; Zhu et al. 2009). Since the Puf2p binding site possesses two copies of the UAAU sequence, the protein may bind as a dimer. Similarly, C. elegans PUF-5 binds UGURYYYUGU, with two copies of the UGU sequence at each end of the core recognition site, and is analogous in that respect (Stumpf et al. 2008a). In neither instance do we detect any evidence of sequential binding of two monomers in gel shift studies, but the proteins could exist as homodimers in solution.

A linker length of $3 \mathrm{nt}$ between the two UAAU elements is preferentially enriched among the mRNAs immunopurified with Puf $2 \mathrm{p}\left(P\right.$-value of $10^{-48}$ vs. $5 \times 10^{-18}$ for a gap of $2 \mathrm{nt}$ and $7 \times 10^{-14}$ for the empty gap; two and zero are the most enriched gap lengths after three) (Fig. 1B). Similarly, the linker sequence AAU was enriched among those motifs containing a 3-nt linker. However, the presence of the linker element had only a marginal effect on binding of Puf2p, as did variations in its length or the identity of the linker nucleotides. The enrichment of a 3-nt linker and the AAU sequence among mRNAs associated with Puf2p and Puflp in vivo likely reflect additional biological functions beyond binding of these proteins. We suggest that the linker spaces between two sets of contacts with UAAU elements. This situation may be analogous to that seen in other PUF proteins. For example, several PUF proteins "flip" bases away from the protein (see Introduction). The identities of flipped bases can vary without affecting binding. We suggest two models of Puf2 $p$ binding. In the first, a single Puf $2 \mathrm{p}$ molecule binds the dual UAAU element, and linker nucleotides in the complexes are flipped away from the protein. In the second, two molecules of Puf2p bind, each one interacting with a single UAAU.

We identified the Puf2p motif in 122 genes of the approximately 5000 analyzed $S$. cerevisiae genes $(<3 \%)$, yet $>50 \%$ of these ( 69 genes) appeared among the mRNAs that are highly associated with Puf2p (top 336 genes). GO term analysis for these 69 genes revealed enrichment for genes encoding membrane proteins $(P$-value $=0.001)$. This enrichment, however, is not necessarily due to the presence of a UAAU motif in their 3' UTRs, because similar enrichment is observed also for Puf2p-associated mRNAs that do not have the UAAU motif. 3' UTRs with multiple occurrences of the UAAU motif tend to be ranked higher (e.g., 11 of the 12 genes with more than one motif are ranked among the top 336 genes). The presence of more than one motif in the PMP2 3' UTR may explain why mutations disrupting only one dual UAAU motif did not completely abolish Puf2p association (Fig. 4B). Multiple iterations of binding sites within $3^{\prime}$ UTRs is a well-established contributor to RBPRNA interactions (Macdonald and Struhl 1988; Kislauskis et al. 1994; Chen and Shyu 1995; Deshler et al. 1997; Loya et al. 2008). Interestingly, DRIM analysis revealed enrichment of the dual UAAU motif also in 5' UTR and ORF regions of the mRNA. Most of the mRNAs with a binding site within their $5^{\prime}$ UTR or ORF do not have a binding site within their $3^{\prime}$ UTR. While the $P$-values were lower than in the $3^{\prime}$ UTR and binding was not verified experimentally, these results further exemplify the complexity and diversity of Puf protein binding.

Puflp appears to have a sequence specificity similar to that of Puf2p, based on both computational analysis of the RNAs with which it associates, as well as direct three-hybrid tests. In immunopurification/microarray studies (Gerber et al. 2004), 90\% (36 of 40) of mRNAs bound to Puflp are also bound by Puf $2 \mathrm{p}$, consistent with the overlap in their specificities. However, $75 \%$ of the mRNAs that bind Puf $2 p$ do not detectably associate with Puflp. If the immunopurification results reflect the in vivo situation, it would imply that additional factors contribute to the specificity of Puf2p 
and enable it to bind those mRNAs. Those might include differences in RNA binding preferences that we have not detected, or collaboration with other protein factors.

In summary, Puf2p and Puflp bind RNA elements that differ from other described PUF binding sites. The nature of its recognition by Puf2p and, specifically, which bases and Puf2p repeats are important for binding will require structural analysis. Regardless, the Puf2p-RNA interaction expands the repertoire of PUF proteins and diversifies their array of RNA specificities.

\section{MATERIALS AND METHODS}

\section{Computational analysis of sequences (DRIM)}

DRIM (discovery of rank imbalanced motifs) is a software application that identifies sequence motifs in lists of ranked DNA and RNA sequences (Eden et al. 2007; http://bioinfo.cs.technion.ac.il/drim/; the $\mathrm{mHG}$ statistics to discover motifs that are statistically enriched at the top of a ranked list of sequences). This statistical approach uses threshold optimization in this ranked list. Briefly, the flexible threshold process computes a hypergeometric tail (HGT) at each possible cutoff of the ranked list and then optimizes the threshold to yield the most significant enrichment of the motif under consideration. Exact $P$-values are obtained as described in Eden et al. (2007). Because DRIM was originally developed to identify enriched motifs in transcription factor target sequences (Eden et al. 2007), the algorithm first had to be adapted to search for enriched motifs in RNA sequences as well. One important feature of the new version of DRIM is the option to differentially weight multiplicities of motif occurrences. New features of the RNA version of DRIM are described at http://bioinfo.cs.technion.ac.il/drim/.

We applied DRIM to analyze a list consisting of 4997 S. cerevisiae 3' UTR sequences, ranked according to Puf2 binding, as reported by Gerber et al. (2004). The yeast 3' UTR sequences were obtained from UCSC ( $S$. cerevisiae genes), according to their experimentally defined lengths (Nagalakshmi et al. 2008). The motifs reported herein were thus found to be enriched in the UTRs ranked at the top of this experimentally derived list.

\section{Protein purification}

The PUM domain of Puf2p (amino acids 361-832) was cloned into pGex6P1 as a GST fusion protein with a $6 \times$ HIS tag, and transformed to BL-21 Escherichia coli cells. Bacteria (0.5 L) were grown on $\mathrm{LB}$ to logarithmic phase $\left(\mathrm{OD}_{600}=0.6-0.9\right)$ and induced for protein expression by adding $1 \mathrm{mM}$ IPTG and growing the cells overnight at $16^{\circ} \mathrm{C}$. Cells were harvested by centrifugation and washed with $50 \mathrm{mM}$ Tris- $\mathrm{HCl}$ at $\mathrm{pH} 8.0$ and $10 \%$ sucrose. Cell pellets were frozen in liquid nitrogen and resuspended in $25 \mathrm{~mL}$ of Lysis Buffer (20 mM HEPES-KOH at $\mathrm{pH} 7.4,0.5 \mathrm{M} \mathrm{NaCl}, 5 \mathrm{mM}$ DTT, $0.02 \%$ Tween 20, 1 mM PMSF, $1 \mu \mathrm{g} / \mathrm{mL}$ leupeptin, $1 \mu \mathrm{g} / \mathrm{mL}$ Pepstatin). The cells were lysed with lysozyme (final concentration of $0.5 \mathrm{mg} / \mathrm{mL}$ ) for $30 \mathrm{~min}$ on ice and were frozen in liquid nitrogen. After thawing the cells, $2 \mathrm{mM} \mathrm{MgCl}_{2}$ and $10 \mathrm{mg} / \mathrm{mL}$ DNase I were added and incubated for $30 \mathrm{~min}$ on ice. The lysate was frozen in liquid nitrogen, thawed, and clarified by centrifugation at 18,000 rpm for $40 \mathrm{~min}$ at $4^{\circ} \mathrm{C}$. Because the Puf2-PUM domain was insoluble and found to accumulate in inclusion bodies, the pellet that contains Puf2-PUM was dissolved in Binding buffer (6 M urea, $15 \mathrm{mM}$ imidazole, $0.5 \mathrm{M} \mathrm{NaCl}, 20 \mathrm{mM}$ Tris- $\mathrm{Cl}$ at $\mathrm{pH} \mathrm{8)}$ and subjected to centrifugation at $18,000 \mathrm{rpm}$ for $40 \mathrm{~min}$ at $4^{\circ} \mathrm{C}$. The supernatant, which contains the dissolved proteins, was filtered through a $0.45-\mu \mathrm{m}$ filter and loaded on an Ni-NTA Agarose bead column pre-equilibrated with Binding buffer. Proteins were eluted with $6 \mathrm{~mL}$ of Elution buffer and were dialyzed into decreasing concentration of urea in $1 \times$ PBS for the refolding process. Proper refolding was assessed by the ability of the GST moiety to bind glutathione Sepharose resin. Purity and concentration of the protein were estimated by SDS gel electrophoresis.

The PUM domain of Puf5p (amino acids 126-626) was cloned into pGex6P1. GST-Puf5p protein purification was performed by the same method described above except that after the centrifugation $\left(18,000 \mathrm{rpm}\right.$ for $40 \mathrm{~min}$ at $\left.4^{\circ} \mathrm{C}\right)$ the supernatant, which includes the protein, was passed through glutathione Sepharose resin (preequilibrated with Lysis buffer). The proteins were eluted from the glutathione Sepharose resin with Elution buffer, and protein purity and concentration were estimated by SDS gel electrophoresis.

\section{EMSA}

RNA oligoribonucleotides (40 pmol; Dharmacon) were end-labeled with $\left[\gamma_{-}{ }^{32} \mathrm{P}\right]$ ATP by T4 polynucleotide kinase for $1 \mathrm{~h}$ at $37^{\circ} \mathrm{C}$ and purified with G-50 columns. For binding reaction, $20 \mathrm{fmol}$ of labeled RNA was incubated for $1 \mathrm{~h}$ at room temperature with varying concentrations of GST-Puf2 or GST-Puf5 in EMSA binding buffer (Koh et al. 2009). Loading dye (6\% glycerol, $0.06 \%$ Bromophenol Blue) was added to each reaction, and samples were loaded on a pre-run, nondenaturing $5 \%$ polyacrylamide gel (BioRad). Gels were resolved in $1 \times$ TBE buffer at $100 \mathrm{~V}$ for $35 \mathrm{~min}$ at $4^{\circ} \mathrm{C}$ and were exposed to a PhosphorImager screen. Signals were quantified with the TINA program. Apparent $K_{\mathrm{d}}$ values were calculated using GraphPad Prism software (version 5.02), by fitting a binding curve to the percentage of bound RNA (which was calculated from densitometry readings of the shifted and unshifted RNA in each reaction) against the protein concentration. Apparent $K_{\mathrm{d}}$ values are represented as an average of two to four repetitions, with standard errors shown in Table 1.

\section{Constructs for yeast three-hybrid assay}

A cDNA encoding $S$. cerevisiae Puf2p (GenBank accession number NM_001184139), comprising amino acids 1-1016, was cloned into an activation domain vector, pGADT7. The PUF repeat mutants used in Figure 5 were created by site-directed mutagenesis. Amino acid changes were as follows: Puf2p R1/R2 mut, N591A, T592A, T627A, and W628A; Puf2p R1/R2/R3 mut, N591A, T592A, T627A, W628A, N664A, and Y665A. For specificity testing, cDNAs encoding S. cerevisiae Puflp (NM_001181748) amino acids 1-1091, Puf3p (NM_001181833) amino acids 511-879, Puf4p (NM_001180879) amino acids 534-888, and Puf5 (NM_001181043) amino acids 26860 were each cloned into pGADT7.

A 27-nt region of the PMP2 3' UTR was cloned into the XmaI and SphI sites of the p3HR2 vector (Stumpf et al. 2008a). The 23rd nucleotide in that region was changed from $U$ to $A$ to avoid five consecutive uridines that will terminate RNA transcription by Pol III (Stumpf et al. 2008b). All other PMP2 RNA constructs were created the same way. The Puf3p binding site B of COX17 3' UTR and the first $50 \mathrm{nt}$ of the $\mathrm{HO}^{\prime}$ UTR were similarly cloned into the p3HR2 vector. 


\section{Yeast three-hybrid assay}

Three-hybrid assays were performed in the YBZ-1 yeast strain as described previously (Hook et al. 2005) with the following modifications: $50 \mu \mathrm{L}$ of a saturated culture was diluted in $1 \mathrm{~mL}$ of selective media and allowed to grow for $3 \mathrm{~h}$ to reach an $\mathrm{OD}_{660}$ of 0.1 to 0.2 . $\beta$-Galactosidase activity was normalized to an $\mathrm{OD}_{660}$ of 0.1 ; values are represented as an average of three repetitions, with standard deviations shown in each figure.

\section{Yeast protein extracts and Western blotting}

The relative expression levels of Puf2p protein in the yeast threehybrid assay were determined using Western blot analysis. Yeast were transformed with the pGADT7-Puf2p (amino acids 1-1016) plasmid as well as the RNA expression plasmid p3HR2. Cultures were grown to saturation in selective media at $30^{\circ} \mathrm{C}$ and then diluted into fresh selective media and grown for $4 \mathrm{~h}$ at $30^{\circ} \mathrm{C}$. One milliliter of log-phase culture was pelleted, resuspended in $100 \mu \mathrm{L}$ of ESB (2\% SDS, $80 \mathrm{mM}$ Tris at pH 6.8, 10\% glycerol, 1.5\% DTT), heated for $3 \mathrm{~min}$ to $100^{\circ} \mathrm{C}$, and then lysed using glass beads and vortexing for $5 \mathrm{~min}$. Extracts were then heated for $1 \mathrm{~min}$ to $100^{\circ} \mathrm{C}$ and stored at $-20^{\circ} \mathrm{C}$.

For Western blot analysis, $10 \mu \mathrm{L}$ of yeast extract from each expression construct was loaded onto an SDS polyacrylamide gel. After electrophoresis, proteins were transferred onto a nitrocellulose membrane and then blocked with TBST + milk ( $1 \times$ TBS, $0.1 \%$ Tween 20, 5\% dry milk [w/v]). To detect the HA-tagged Puf2p fusion proteins, we incubated the blot with HA-11 antibody (Covance) at a 1:1000 ratio in TBST + milk for $1 \mathrm{~h}$ at room temperature. The blot was then washed with TBST $(1 \times$ TBS, $0.1 \%$ Tween 20$)$ three times and then probed with the secondary antibody, HRP-conjugated goat anti-mouse, for $1 \mathrm{~h}$ at room temperature in TBST + milk. After the blot was washed three times with TBST, HRP was detected using the Pierce ECL Western Blotting Substrate kit.

\section{RNA affinity purification}

Exponentially growing cells $(1 \mathrm{~L})$ were collected by centrifugation at $4000 \mathrm{rpm}$ for $4 \mathrm{~min}$ at $4^{\circ} \mathrm{C}$ and resuspended and washed two times in $40 \mathrm{~mL}$ of TAP-Buffer A (20 mM Tris-Cl at $\mathrm{pH} 8,140$ $\mathrm{mM} \mathrm{KCl}, 1.8 \mathrm{mM} \mathrm{MgCl}_{2}, 0.1 \% \mathrm{NP}-40$, and $0.02 \mathrm{mg} / \mathrm{mL}$ heparin). Cells were then resuspended in $5 \mathrm{~mL}$ of TAP-Buffer B (buffer A + $0.5 \mathrm{mM}$ DTT, $1 \mathrm{mM}$ PMSF, $0.5 \mu \mathrm{g} / \mathrm{mL}$ leupeptin, $0.8 \mu \mathrm{g} / \mathrm{mL}$ Pepstatin, $0.2 \mathrm{mg} / \mathrm{mL}$ heparin, $100 \mathrm{U} / \mathrm{mL}$ RNasin, and $20 \mathrm{U} / \mathrm{mL}$ DNase I), and aliquots of $1 \mathrm{~mL}$ were transferred to screw-capped microfuge tubes supplemented with $1 \mathrm{~mL}$ of chilled glass beads. Cells were lysed by two rounds of $90-$ sec pulses in a bead beater. The lysate was transferred to a clean microfuge tube and cleared from cell debris by centrifugation at $10,000 \mathrm{rpm}$ for $10 \mathrm{~min}$ at $4^{\circ} \mathrm{C}$. For unprecipitated control ("Input"), one-tenth of the lysate was precipitated and one-tenth was taken for Northern analysis. Affinity purification was performed in $15-\mathrm{mL}$ tubes, by incubating $4.5 \mathrm{~mL}$ of lysate with $400 \mu \mathrm{L}$ of IgG beads (pre-equilibrated with TAP-Buffer A) for $2 \mathrm{~h}$ at $4^{\circ} \mathrm{C}$ with constant rolling. Bound material was washed by four rounds of centrifugation at $3000 \mathrm{rpm}$ at $4^{\circ} \mathrm{C}$ and addition of $4 \mathrm{~mL}$ of ice-cold TAP-Buffer C $(20 \mathrm{mM}$ Tris- $\mathrm{Cl}$ at $\mathrm{pH} 8,140 \mathrm{mM} \mathrm{KCl}, 1.8 \mathrm{mM} \mathrm{MgCl}_{2}, 0.01 \% \mathrm{NP}-40,0.5$ $\mathrm{mM}$ DTT, $10 \mathrm{U} / \mathrm{mL}$ RNasin). Proteins and RNA were eluted by TEV-protease cleavage for $2 \mathrm{~h}$ at $16^{\circ} \mathrm{C}$ in a TEV reaction buffer (provided by the manufacturer). The eluted RNA was extracted by phenol-chloroform, subjected to ethanol precipitation, and resuspended in $50 \mu \mathrm{L}$, and one-third was subjected to Northern analysis (Eldad et al. 2008).

\section{SUPPLEMENTAL MATERIAL}

Supplemental material is available for this article.

\section{ACKNOWLEDGMENTS}

We thank Gal Shaul and Naama Hazan for their role in developing DRIM for RNA and for data analysis. We thank Israel Steinfeld for providing access to $\mathrm{mHG}$ implementation. We also are grateful for the assistance of Laura Vanderploeg with the figures. We appreciate help and discussions from the members of the Arava and Wickens laboratories. This work was supported by ISF grant number 1193/ 09 to Y.A., NIH grants GM32892 and GM50942 to M.W., the $\mathrm{A}^{\star}$ STAR Program (to Y.K.), an NIH Genetics Training Grant (to J.C.), and the Google Anita Borg Memorial Scholarship (to L.L.).

Received March 1, 2011; accepted May 5, 2011.

\section{REFERENCES}

Bailey TL, Boden M, Buske FA, Frith M, Grant CE, Clementi L, Ren J, Li WW, Noble WS. 2009. MEME SUITE: tools for motif discovery and searching. Nucleic Acids Res 37: W202-W208.

Bernstein D, Hook B, Hajarnavis A, Opperman L, Wickens M. 2005. Binding specificity and mRNA targets of a C. elegans PUF protein, FBF-1. RNA 11: 447-458.

Chen CY, Shyu AB. 1995. AU-rich elements: characterization and importance in mRNA degradation. Trends Biochem Sci 20: 465-470.

Clery A, Blatter M, Allain FH. 2008. RNA recognition motifs: boring? Not quite. Curr Opin Struct Biol 18: 290-298.

Deng Y, Singer RH, Gu W. 2008. Translation of ASH1 mRNA is repressed by Puf6p-Fun12p/eIF5B interaction and released by CK2 phosphorylation. Genes Dev 22: 1037-1050.

Deshler JO, Highett MI, Schnapp BJ. 1997. Localization of Xenopus Vg1 mRNA by Vera protein and the endoplasmic reticulum. Science 276: 1128-1131.

Eden E, Lipson D, Yogev S, Yakhini Z. 2007. Discovering motifs in ranked lists of DNA sequences. PLoS Comput Biol 3: e39. doi: 10.1371/journal.pcbi.0030039.

Edwards TA, Pyle SE, Wharton RP, Aggarwal AK. 2001. Structure of Pumilio reveals similarity between RNA and peptide binding motifs. Cell 105: 281-289.

Eldad N, Yosefzon Y, Arava Y. 2008. Identification and characterization of extensive intra-molecular associations between $3^{\prime}$-UTRs and their ORFs. Nucleic Acids Res 36: 6728-6738.

Eliyahu E, Pnueli L, Melamed D, Scherrer T, Gerber AP, Pines O, Rapaport D, Arava Y. 2010. Tom20 mediates localization of mRNAs to mitochondria in a translation-dependent manner. Mol Cell Biol 30: 284-294.

Galgano A, Forrer M, Jaskiewicz L, Kanitz A, Zavolan M, Gerber AP. 2008. Comparative analysis of mRNA targets for human PUF-family proteins suggests extensive interaction with the miRNA regulatory system. PLoS ONE 3: e3164. doi: 10.1371/journal.pone.0003164.

Gerber AP, Herschlag D, Brown PO. 2004. Extensive association of functionally and cytotopically related mRNAs with Puf family RNA-binding proteins in yeast. PLoS Biol 2: e79. doi: 10.1371/ journal.pbio.0020079.

Gu W, Deng Y, Zenklusen D, Singer RH. 2004. A new yeast PUF family protein, Puf6p, represses ASH1 mRNA translation and is required for its localization. Genes Dev 18: 1452-1465. 
Gupta YK, Nair DT, Wharton RP, Aggarwal AK. 2008. Structures of human Pumilio with noncognate RNAs reveal molecular mechanisms for binding promiscuity. Structure 16: 549-557.

Hieronymus H, Silver PA. 2003. Genome-wide analysis of RNAprotein interactions illustrates specificity of the mRNA export machinery. Nat Genet 33: 155-161.

Hogan DJ, Riordan DP, Gerber AP, Herschlag D, Brown PO. 2008. Diverse RNA-binding proteins interact with functionally related sets of RNAs, suggesting an extensive regulatory system. PLoS Biol 6: e255. doi: 10.1371/journal.pbio.0060255.

Hook B, Bernstein D, Zhang B, Wickens M. 2005. RNA-protein interactions in the yeast three-hybrid system: Affinity, sensitivity, and enhanced library screening. RNA 11: 227-233.

Hook BA, Goldstrohm AC, Seay DJ, Wickens M. 2007. Two yeast PUF proteins negatively regulate a single mRNA. J Biol Chem 282: 15430-15438.

Kanitz A, Gerber AP. 2010. Circuitry of mRNA regulation. Wiley Interdiscip Rev Syst Biol Med 2: 245-251.

Keene JD, Tenenbaum SA. 2002. Eukaryotic mRNPs may represent posttranscriptional operons. Mol Cell 9: 1161-1167.

Kershner AM, Kimble J. 2010. Genome-wide analysis of mRNA targets for Caenorhabditis elegans FBF, a conserved stem cell regulator. Proc Natl Acad Sci 107: 3936-3941.

Kislauskis EH, Zhu X, Singer RH. 1994. Sequences responsible for intracellular localization of beta-actin messenger RNA also affect cell phenotype. J Cell Biol 127: 441-451.

Koh YY, Opperman L, Stumpf C, Mandan A, Keles S, Wickens M. 2009. A single C. elegans PUF protein binds RNA in multiple modes. RNA 15: 1090-1099.

Kraemer B, Crittenden S, Gallegos M, Moulder G, Barstead R, Kimble J, Wickens M. 1999. NANOS-3 and FBF proteins physically interact to control the sperm-oocyte switch in Caenorhabditis elegans. Curr Biol 9: 1009-1018.

Loya A, Pnueli L, Yosefzon Y, Wexler Y, Ziv-Ukelson M, Arava Y. 2008. The $3^{\prime}$-UTR mediates the cellular localization of an mRNA encoding a short plasma membrane protein. RNA 14: 1352-1365.

Macdonald PM, Struhl G. 1988. Cis-acting sequences responsible for anterior localization of bicoid mRNA in Drosophila embryos. Nature 336: 595-598.

Miller MT, Higgin JJ, Hall TM. 2008. Basis of altered RNA-binding specificity by PUF proteins revealed by crystal structures of yeast Puf4p. Nat Struct Mol Biol 15: 397-402.

Morris AR, Mukherjee N, Keene JD. 2008. Ribonomic analysis of human Pum1 reveals cis-trans conservation across species despite evolution of diverse mRNA target sets. Mol Cell Biol 28: 4093-4103.

Nagalakshmi U, Wang Z, Waern K, Shou C, Raha D, Gerstein M, Snyder M. 2008. The transcriptional landscape of the yeast genome defined by RNA sequencing. Science 320: 1344-1349.
Olivas W, Parker R. 2000. The Puf3 protein is a transcript-specific regulator of mRNA degradation in yeast. EMBO J 19: 6602-6611.

Opperman L, Hook B, DeFino M, Bernstein DS, Wickens M. 2005. A single spacer nucleotide determines the specificities of two mRNA regulatory proteins. Nat Struct Mol Biol 12: 945-951.

Saint-Georges Y, Garcia M, Delaveau T, Jourdren L, Le Crom S, Lemoine S, Tanty V, Devaux F, Jacq C. 2008. Yeast mitochondrial biogenesis: A role for the PUF RNA-binding protein Puf3p in mRNA localization. PLoS ONE 3: e2293. doi: 10.1371/journal. pone.0002293.

Scherrer T, Mittal N, Janga SC, Gerber AP. 2010. A screen for RNAbinding proteins in yeast indicates dual functions for many enzymes. PLoS ONE 5: e15499. doi: 10.1371/journal.pone.0015499.

Sonoda J, Wharton RP. 1999. Recruitment of Nanos to hunchback mRNA by Pumilio. Genes Dev 13: 2704-2712.

Stumpf CR, Kimble J, Wickens M. 2008a. A Caenorhabditis elegans PUF protein family with distinct RNA binding specificity. RNA 14: 1550-1557.

Stumpf CR, Opperman L, Wickens M. 2008b. Analysis of RNAprotein interactions using a yeast three-hybrid system. Methods Enzymol 449: 295-315.

Tsvetanova NG, Klass DM, Salzman J, Brown PO. 2010. Proteomewide search reveals unexpected RNA-binding proteins in Saccharomyces cerevisiae. PLoS ONE 5: e12671. doi: 10.1371/journal.pone. 0012671.

Ulbricht RJ, Olivas WM. 2008. Puflp acts in combination with other yeast Puf proteins to control mRNA stability. RNA 14: 246-262.

Wang X, Zamore PD, Hall TM. 2001. Crystal structure of a Pumilio homology domain. Mol Cell 7: 855-865.

Wang X, McLachlan J, Zamore PD, Hall TM. 2002. Modular recognition of RNA by a human Pumilio-homology domain. Cell 110: $501-512$.

Wang Y, Opperman L, Wickens M, Hall TM. 2009. Structural basis for specific recognition of multiple mRNA targets by a PUF regulatory protein. Proc Natl Acad Sci 106: 20186-20191.

Wickens M, Bernstein DS, Kimble J, Parker R. 2002. A PUF family portrait: 3'UTR regulation as a way of life. Trends Genet 18: 150157.

Zamore PD, Williamson JR, Lehmann R. 1997. The Pumilio protein binds RNA through a conserved domain that defines a new class of RNA-binding proteins. RNA 3: 1421-1433.

Zhang B, Gallegos M, Puoti A, Durkin E, Fields S, Kimble J, Wickens MP. 1997. A conserved RNA-binding protein that regulates sexual fates in the C. elegans hermaphrodite germ line. Nature 390: 477484.

Zhu D, Stumpf CR, Krahn JM, Wickens M, Hall TM. 2009. A 5' cytosine binding pocket in Puf3p specifies regulation of mitochondrial mRNAs. Proc Natl Acad Sci 106: 20192-20197. 

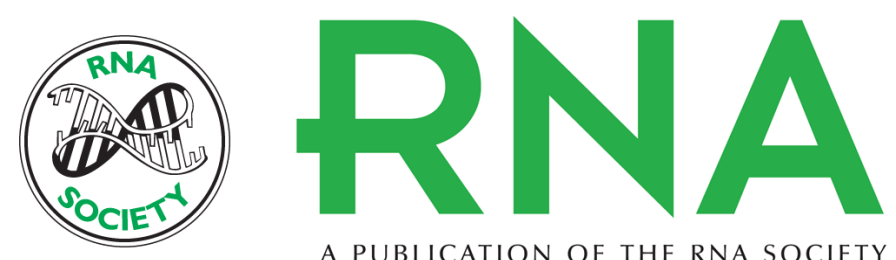

A PUBLICATION OF THE RNA SOCIETY

\section{Divergent RNA binding specificity of yeast Puf2p}

Yahav Yosefzon, Yvonne Y. Koh, Jacqueline J. Chritton, et al.

RNA 2011 17: 1479-1488 originally published online June 17, 2011

Access the most recent version at doi:10.1261/rna.2700311

Supplemental

Material

References This article cites 45 articles, 20 of which can be accessed free at:

http://rnajournal.cshlp.org/content/17/8/1479.full.html\#ref-list-1

\section{License}

Email Alerting

Service

http://rnajournal.cshlp.org/content/suppl/2011/06/01/rna.2700311.DC1

top right corner of the article or click here. 